\title{
Linking biomedical research to health care
}

W e are in the midst of a vitally important discussion on health care. This debate has provided insights into who we are as Americans and what we value. We clearly value our health and our health care. We believe that health and access to quality health care are a human right. As physicians, many of us have worked in impoverished areas in this country and abroad, and we've seen that the poor can often be deprived of such rights. We have had the privilege of providing medical care to people who otherwise would not be able to get it. The number of health care professionals eager to serve is impressive, and so is the amount that can be accomplished. And so, our efforts as physicians to advocate for health as a human right, to reduce Americans' vulnerability, and to care for those in need must be heard.

Another insight is obvious to those who engage in biomedical research, but the message is likely underappreciated by the American public. Biomedical research provides the basis for progress in health and health care. Basic discoveries, translation to clinical medicine, and implementation into medical practice have been the story line of medical advances for decades. And the vast majority of basic discoveries have been funded by the National Institutes of Health through taxpayers' investment in biomedical research. So why isn't NIH-funded research mentioned more in our health care discussion? We know the likely causes: it is difficult to draw a straight line between NIH-funded research and improvements in health care; many basic science advances take decades before being fully developed into therapeutics and fully implemented into medical practice; and reporting of medi- cal advances rarely includes attribution to NIH funding. But this is a very special time for our biomedical community. We are the recipients of approximately $\$ 10$ billion of American Recovery and Reinvestment Act (ARRA) funds, and we can use the health care discussion as an opportunity to articulate (a) our gratitude for the enormous investment made

\section{The physician's voice}

Knowledge gained from important fields of $\mathrm{NIH}$-funded research will contribute significantly to our progress in achieving affordable and high-quality health care for all Americans.

by the American public in the biomedical research enterprise; (b) how ARRA-supported research will accelerate medical progress by helping to deliver new treatments and prevention strategies to reduce disease; (c) the synergy among university, academic health centers, and NIH support for training the next generation of physician-scientists and biomedical leaders; and (d) the current and future scientific opportunities that will empower the biomedical research community.

Knowledge gained from important fields of NIH-funded research will contribute significantly to our progress in achieving affordable and high-quality health care for all Americans. Comparative effectiveness research, for example, involves conducting and synthesizing research comparing the benefits and harms of different interventions and strategies to prevent, diagnose, treat, and monitor health conditions in real-world settings. The goal of this research is to improve health outcomes by developing and disseminating evidence-based information to patients, clinicians, and other decision makers about which interventions are most effective for which patients in specific circumstances. The NIH has a long and rich tradition of comparative effectiveness research and is well positioned to play a major leadership role in continuing to fund this research. We understand that patient-centered health research is vital to effective health care, and the results of comparative effectiveness research can direct the right treatment to the right person in the right setting at the right time. Other areas in which NIH-funded research will inform the health care discussion include health economics, health systems research, health disparities, and personalized medicine, to name a few.

As physicians, we carry within us a belief that health and access to quality health care are a human right; that when the health system does not serve many of our fellow Americans well, we must change it. Biomedical research offers hope to improve vaccines, therapeutics, devices, and health system approaches that will bring health and security to our fellow Americans. Our health care discussion is not solely about our nation's health; it is also a statement about what we value.

\section{Elizabeth G. Nabel}

National Heart, Lung, and Blood Institute, Bethesda, Maryland, USA. E-mail: directornhlbi@mail.nih.gov.

J. Clin. Invest. 119:2858 (2009). doi:10.1172/ JCI41035.

\section{Reflections on health care}

W hat do we need? Not what we have: a health care system whose costs are spiraling out of control, that fails to provide care for almost 50 million Americans, and that ranks far from the top in terms of infant mortality and adequate health care for our adult population. Ideally, we need a single- payer, universal health care structure. To paraphrase Ted Kennedy, why shouldn't all Americans have access to the kind of health care that is offered to our congressmen and senators? Failing that, health insurance companies should go away or agree to compete on an equal footing with govern- ment-sponsored health care in a truly free market economy.

Built into that system, though, must be the recognition that we cannot afford to spend such a large proportion of our health care dollars on the final few months of life. It is agonizing to watch hundreds of thou- 